Presented at the 1978 . IEEE Nuclear Science

Symposium, Washington, D. C., 0ctober 18-20,

\title{
THEORY OF IMAGING WITH A VERY LIMITED \\ NUMBER OF PROJECTIONS
}

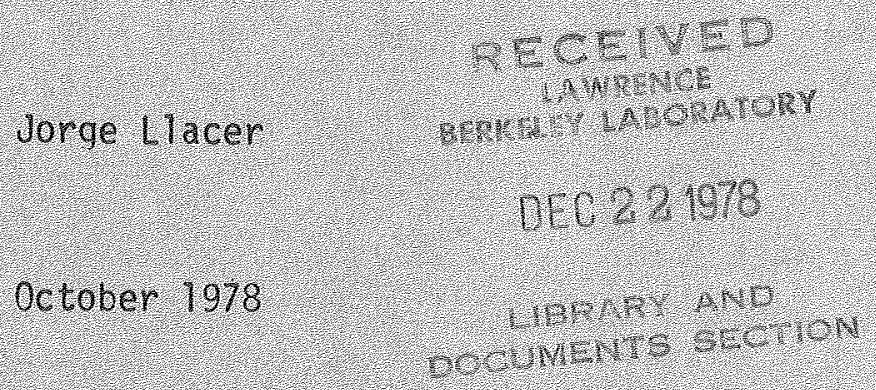

Prepared for the I. S. Department of Eneray under Contract W-7405-ENG-48

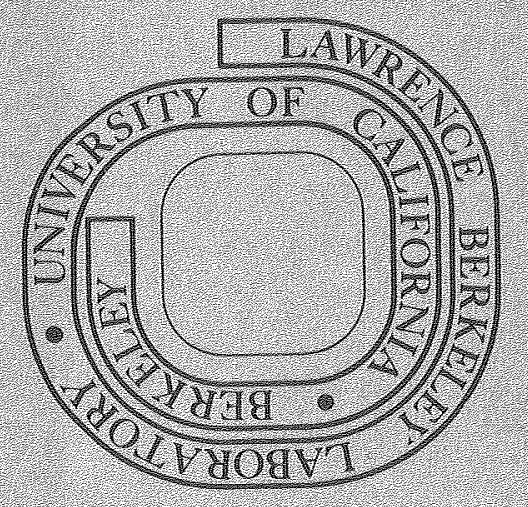

\section{TWO-WEEK LOAN COPY}

This is a Library Circulating Copy which may be borrowed for two weeks. For a personal retention copy, call Tech. Info. Division, Ext. 6782 


\section{LEGAL NOTICE}

This report was prepared as an account of work sponsored by the United States Government. Neither the United States nor the Departmeni of Energy, nor any of their employees, nor any of their coniractors, subcontractors, or their employees, makes any warranty, express or implied. the accuracy, connoleteness or usetutness of any information, apparatus, product or process disclosed, or represents that its use would not infringe privately, nwed rights.

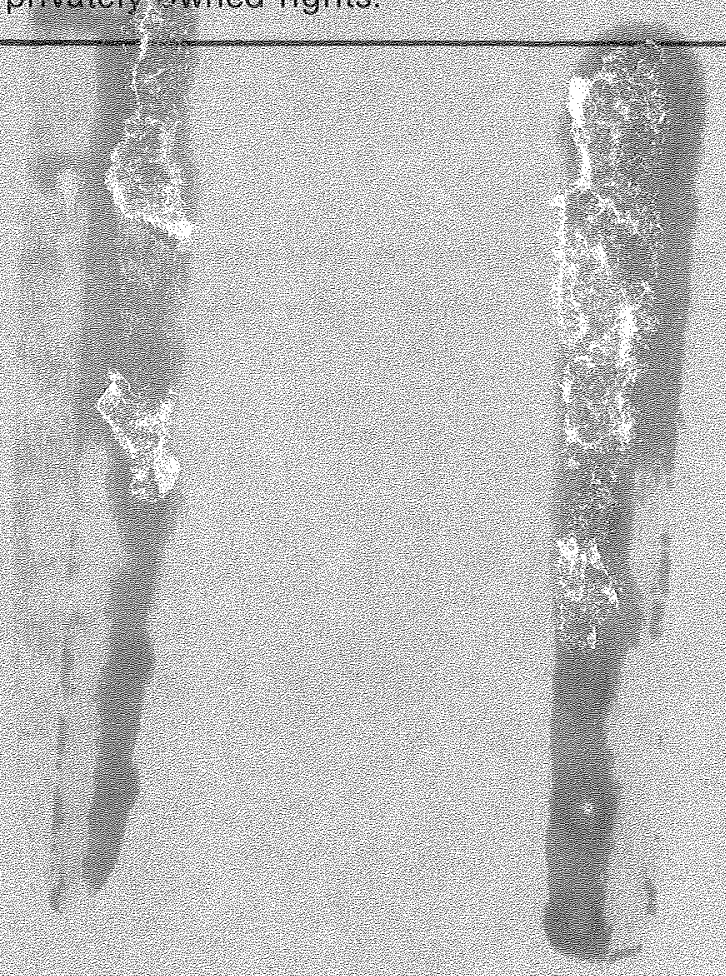


Jorge L7acer

Lawrence Berkeley Laboratory

University of California

Berkeley, California 94720

\section{Abstract}

A theory of imaging for detector systems with a very 7 imited number of projections has been developed. The relationships between a matrix which determines the system, its eigenvectors and eigenvalues, and the physical characteristics of the detector system are analyzed in order to assist in the most effective design of an instrument. It is shown that reconstruction methods for complete data sets are essentially an extension of the methods deveToped for incomplete sets. The concept of mathematical sweeping to replace mechanical detector motion in incomplete detector systems is demonstrated.

\section{Introduction}

The theory of three dimensional image reconstruction from projections is well established for the cases in which radiation source and detector configurations allow a complete set of data to be obtained. A complete set of data could be described as a sufm ficient number of line projections at a sufficient number of angular increments such that enough independent measurements are made to allow the image reconstruction of a complete bound region. The accuracy and definition of the reconstruction will then depend principally on the spacing between line projections. the size of the angular increments, the amount of crosstalk or overlap between detectors, the statistics of the measurement, and on the algorithms used for the reconstruction. A good review of reconstruction methods is given in Ref. 1.

In cases where the imaging instrument is conm strained by cost, detector efficiency requirements or other considerations to a design that cannot generate a complete set of data covering the volume to be observed, the available algorithms cannot be used for reconstruction. That situation would arise, for example, in the design of an instrument to image very small amounts of positron emitier activity in a cylindrical volume, in which a high efficiency and low cost requirement determine the use of a sma17 number of relatively large detectors and operational requirements define a geometry which is not a complete cylindrical ring.

In the conventional language of image reconstruction, the detector elements of such 1 imited systems would have large dimensions compared to the desired separation between sampling points, and the number of projections that can be obtained from the non-rotating instrument is much too small for a conventional Fourier-based reconstruction algorithm. Another very important characteristic of these systems is that the point response function may not be space invariant even within a single image plane; that is, moving a point source from $r_{i}$ to $r_{i}+j$ does not necessarily form an identical pattern of detector responses displaced at the detector plane by a distance proportional to $\left|r_{i}-r_{i}+j\right|$. Under these conditions,

*This work was supported by the Division of Biomedical and Environmental Research of the Department of Energy under Contract No. W-7405-ENG-48. solutions to the imaging problem by deconvolution are not feasible. For these very limited systems, we are left then with purely algebraic methods of image reconstruction, which will approach the more conventional methods as the number of projections and angles increases towards a complete set of data.

The purpose of this paper is to develop the theory of image reconstruction for systems giving incomplete sets of data, and to relate the mathematical results to the characteristics of detector dimensions and location for the purpose of obtaining the best possible image within a given set of constraints.

\section{The System Matrix}

Let us consider a general array of detectors with $n$ outputs; for example, in a coincidence annihilation radiation detector there could be $n$ possible chords joining pairs of crystals. If we place a point source during a suitable fixed length of time at position $s_{j}$ in a set of m source positions, the detectors will respond with a vector of $n$ elements, each element conm taining the number of times that a detector response has occurred. If this experiment is repeated for all m source positions and the resulting vectors are set side by side as column vectors, we wit7 obtain a system matrix $A$ with $n$ rows (one per possible detector out put) and $m$ columns (one for each source position). The m source positions used to obtain the A matrix will be called "system points." With this matrix we can, in principle, solve the imaging problem

$$
A \vec{x}=\vec{k}
$$

where $\vec{k}$ is a vector of detector responses caused by unknown amounts of activity $\vec{x}$ placed at the system point $s_{j}$

If $n=m, E q .1$ could be solved directly if $A$ is not singular and of small enough size that truncation errors do not accumulate adversely. If $n \neq m$, the solution to Eq. 1 is equivalent to solving

$$
A^{\top} A \vec{x}=A^{\top} \vec{k}
$$

where $A^{\top}$ is the transpose of $A$. Defining $A^{\prime}=A^{\top} A$ and $k^{\prime}=A^{T} \vec{k}$, we rewrite $E q .2$ as

$$
A^{\prime} \vec{x}=\vec{k}^{\prime}
$$

where $A^{\prime}$ is (mxm), and $\vec{x}$ and $\vec{k}^{\prime}$ are also of dimension $m$ (the number of system points).

The idea of solving Eqs. 1 or 3 directly is an old one, and has been reported by Robertson, Marr, Rosenblum, Radeka and Yamamoto ${ }^{2}$ to lead to consider able error during the inversion process with experimental data in their 32-crystal, positron-annihilation ring detector. 


\section{Eigenvalue Analysis}

We proceed now to examine the problem in terms of the eigenvectors and eigenvalues of the matrix $A^{\prime}$. From the theory of matrices, we know that $A^{\prime}$ is symmetric and real, and that, for this reason, there exists a transformation $\mathrm{H}$ such that

$$
H^{-1} A^{\prime} H=\operatorname{diag}\left(\lambda_{j}\right)=0
$$

where $D$ is a matrix with values $\lambda_{j}$ in the diagonal elements, the eigenvalues of $A^{\prime}$, and all other elements zero.

The matrix $H$ of the transformation has columns that form the eigenvectors of $A^{\prime}$. In the present case, these eigenvectors $\vec{x}_{j}$ form an orthonormal basis for the space spanned by $A^{\prime}$ so that the solution $\vec{x}$ to Eq. 3 can be expanded in that basis to

$$
\vec{x}=\zeta_{1} \vec{x}_{1}+\zeta_{2} \vec{x}_{2}+\ldots+\zeta_{m} \vec{x}_{m}
$$

Since eigenvectors and eigenvalues are defined by the equation

$$
\left(A^{\prime}-\lambda_{j} I\right) \vec{X}_{j}=0
$$

where I is the identity matrix, it follows from Eqs. 3,5 and 6 that

$$
A^{\prime} \vec{x}=\zeta_{1} \lambda_{1} \vec{x}_{1}+\zeta_{2} \lambda_{2} \vec{x}_{2}+\ldots \zeta_{m} \lambda_{m} \vec{x}_{m}=\vec{k}^{\prime}
$$

similarly the experimental result $k^{\prime}$ can be expanded as

$$
\vec{k}^{\prime}=\alpha_{1} \vec{x}_{1}+\alpha_{2} \vec{x}_{2}+\ldots+\alpha_{m} \vec{x}_{m}
$$

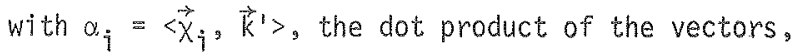
because of orthogonality of the $\vec{x}^{\prime}$ s. Then, equating Eqs. 7 and 8 , we can find

$$
\zeta_{i}=\frac{\alpha_{i}}{\lambda_{i}} \text { for } 1 \leq i \leq m
$$

and finally the solution to Eq. 3 is given by

$$
\vec{x}=\frac{\alpha_{1}}{\lambda_{1}} \vec{x}_{1}+\frac{\alpha_{2}}{\lambda_{2}} \vec{x}_{2}+\ldots+\frac{\alpha_{m}}{\lambda_{m}} \vec{x}_{m} \text {. }
$$

Because the eigenvectors are all of unit length, it is clear that in the presence of fluctuations in the experimental results (transmitted to the values of $\left.\alpha_{i j}\right)$, the resultant vector $\vec{x}$ will be meaningful only if there are no terms in Eq. 10 with values of $\lambda_{i}$ that are much smaller than the rest. If this were the case, a small error in the corresponding value of $\alpha_{i}$ would result in a greatly exaggerated contribution of $\vec{x}_{\dot{j}}$ to the resulting vector $\vec{x}$.

This result is a fundamental property of the solution. Limitations of the chosen method of solu tion, truncation errors of the computer, etc., will only aggravate the problem. One simple measure of the invertibility of a matrix with respect to statistical fluctuations is given by the "condition number" of the matrix, ${ }^{3}$
Condition number $=\lambda_{\max } / \lambda_{\min }$,

which is suitably normalized by the numerator. Evidently, a large condition number is undesirable.

A very useful concept emerges from the above analysis. Large condition numbers result from a sem lection of system points in which two or more are too close to each other so that the detector responses do not define the point unambiguously.

In order to illustrate the validity of the above statement, let us consider an imaging system formed by a number of rings of detectors stacked in the form of a cylinder with system points located at the geom metrical center of each ring only, as in Fig. 1a for a three-ring system. A simple geometrical construction shows that the set of coincidences generated from each system point is totally different from the sets of coincidences from the other points with no coincidences belonging to more than one system point. Under these conditions, matrix $A$ will have a configuration of columns of the form, for example,

$\begin{array}{llll}a_{1,1} & 0 & 0 & 0 \\ a_{2,1} & 0 & 0 & 0 \\ a_{3,1} & 0 & 0 & 0 \\ a_{4,1} & 0 & 0 & 0 \\ 0 & a_{5,2} & 0 & 0 \\ 0 & a_{6,2} & 0 & 0 \\ 0 & a_{7,2} & 0 & 0 \\ 0 & 0 & a_{8,3} & 0 \\ 0 & 0 & a_{9,3} & 0 \\ 0 & 0 & a_{10,3} & 0 \\ 0 & 0 & a_{11,3} & 0 \\ 0 & 0 & a_{12,3} & 0 \\ 0 & 0 & 0 & 0 \\ . & \cdot & . & . \\ . & . & \cdot & \cdot \\ 0 & \cdot & \cdot & a_{n, m} \\ 0 & 0 & 0 & \end{array}$

The system matrix $A^{\prime}=A^{\top} A$ will then be purely diagonal with eigenvectors given by unity vectors,

$$
\begin{aligned}
& 100 \\
& \begin{array}{lll}
0 & 1 & 0
\end{array} \\
& 0,0 \ldots 0 \text {. } \\
& \text {. } \\
& \text { - . } \\
& \begin{array}{lll}
0 & 0 & 1
\end{array}
\end{aligned}
$$

The values of the diagonal elements of $A^{\prime}$ will be the eigenvalues $\lambda_{j}$. 

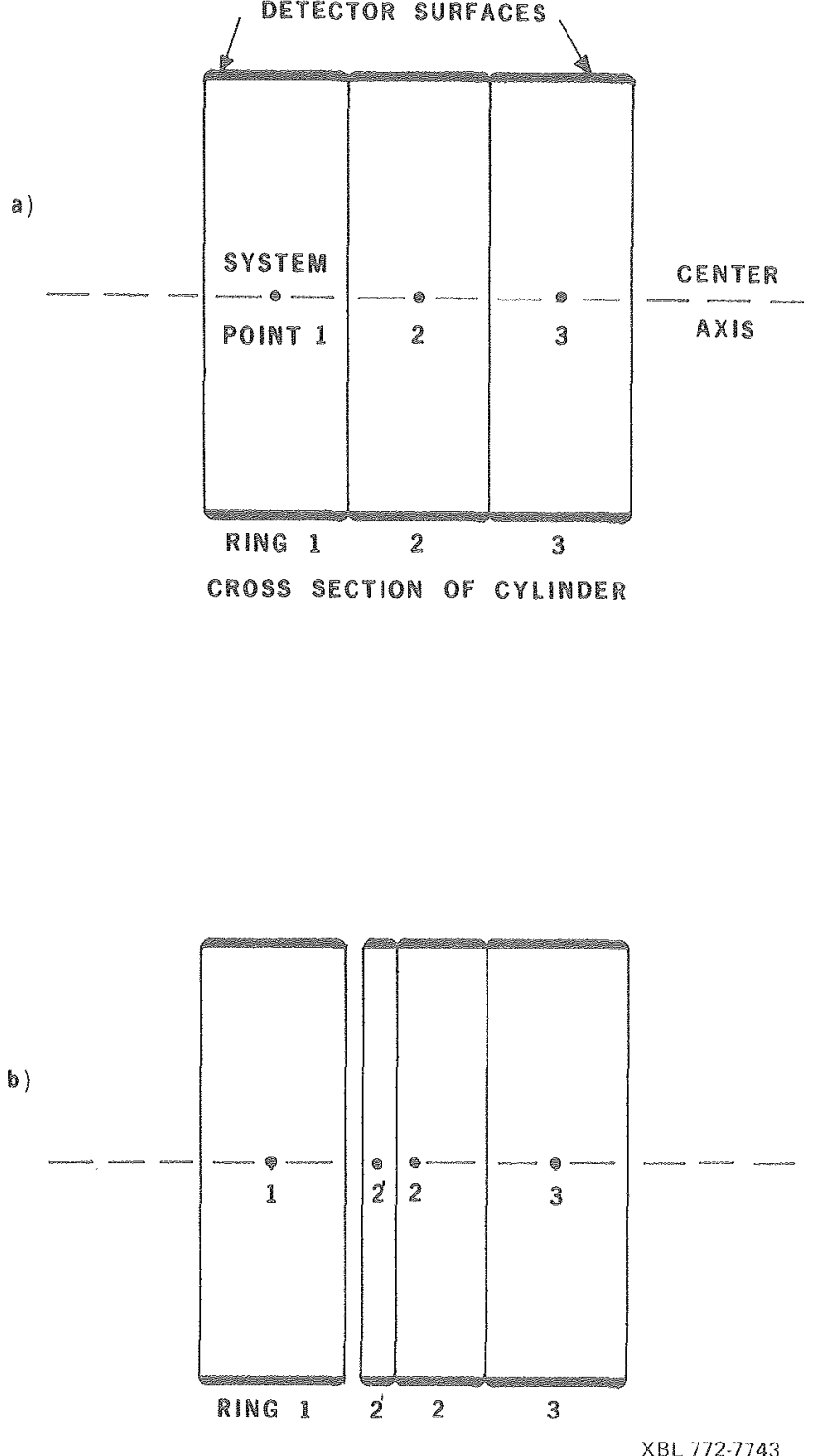

Fig. 1 (a) Cross section of a hypothetical system consisting of three rings of detectors and three system points in the respective centers, resulting in a purely diagonal system matrix $A^{\prime}$. (b) Fourming structure required for a purely diagonal system matrix $A^{\prime}$ when a new point $2^{\prime}$ is added to the above.

If we consider the eigenvalues $\lambda_{j}$ in some detail, we notice that their magnitudes are equal to the sum of the squares of the number of occurrences of all possible coincidences for a particular system point; that is,

$$
\lambda_{j}=\sum_{i}\left(a_{i j}\right)^{2}
$$

This equation indicates that, if one of the points of this simple system is only seen by detectors with small efficiency or by fewer detectors, the eigenvalue corresponding to that point will be smaller than those of the others and lead to a large condition number $\lambda_{\max } / \lambda_{\min }$.
L.BL -8137

In a more general case with system points located arbitrarily, matrix A will contain rows that are often not zero in several columns so that $A^{\prime}$ will not be purely diagonal. It can be diagonalized, however, by a matrix $H$ of eigenvectors ( $\mathrm{Eq} .4$ ) so that the eigenvalues $\lambda_{j}$ of $A^{\prime}$ appedr at the diagonal of $H^{-1} A^{1} H$.

At though the physical interpretation of the eigenvalues is not as simple for the general case as for the one described above, we note now that the eigenvalues of $A^{\prime}$ are the same as those of $\left(H^{-1} A^{\prime} H\right)$, as can be seen by replacing the latter for $A^{:}$in Eq. 6 .

The diagonalization of $A^{\prime}$ by the matrix $H$ is equivatent to a transformation of the detector system so that there should be, at least in concept, one ideal configuration of detectors for any arbitrary set of system points that yields a purely diagonal $A^{\prime}$ matrix with elements $\lambda_{j}$. If the condition number of

this more general system is large, by analogy with the simple case given above, the detector locations of the conceptual "ideal" system are such that one or more of the the system points are seen very little; that is, few coincidences are seen from that point by the detectors. In the real detector systen, this will occur when one system point is located too near another point.

This effect can be readily understood for a very simple case by returning to the example of the stacked rings of detectors with system points at the center of the ring planes as in Fig. 1. The addition of one more system point on the axis of the cylinder very near one of the "good" system points will result in $A^{\prime}$ not being purely diagonal. The diagonalization prom cedure through $H$ will be equivalent to forming a new "ideal" detector system with one more ring as shown in Fig. 1b. The requirement of the uniqueness of the coincidences forces ring $2^{\prime}$ to be much smaller than the others and a reduction in the size of ring 2 as we 71 . System point 2 witl have coincidences between rings 2 and $2^{\prime}$, but point $2^{\prime}$ will have none between the same rings. It is evident that the "ideal" system of Fig. Ib will have a larger condition number than that of Fig. la and that the closer point $2^{\prime}$ is made to 2, the worse the situation will be.

\section{Physical Meaning of Matrix $A$}

The matrix $A^{\prime}$, as described above, is symmetric and it will contain as many rows (or columns) as system points. As we sha!l see below, the contents of each row provides meaningful information as to which specific system points are not sufficiently well sampled by the detectors and/or which points are ambiguously sampled resulting in high condition numbers.

Let us assume that the activity $\vec{x}$ of $E q .1$ is unity at system point $s_{j}$ and zero elsewhere. Vector $\vec{k}, E q .1$, will contain $n$ elements with magnitudes proportional to the rates of all possible coincidences due to that unit activity. Let us consider the $e^{\text {th }}$ elements of vector $\vec{k}, i . e$. , one of the possible coincidences with rate $r_{\ell}$. The contribution of that $\ell^{\text {th }}$ element to vector $\vec{k}^{\prime}=A^{\top} k$ will be a vector $r_{2} \vec{r}_{2}$, where $\vec{r}_{Q}$ is given by the $e^{\text {th }}$ row of the matrix $A$. 

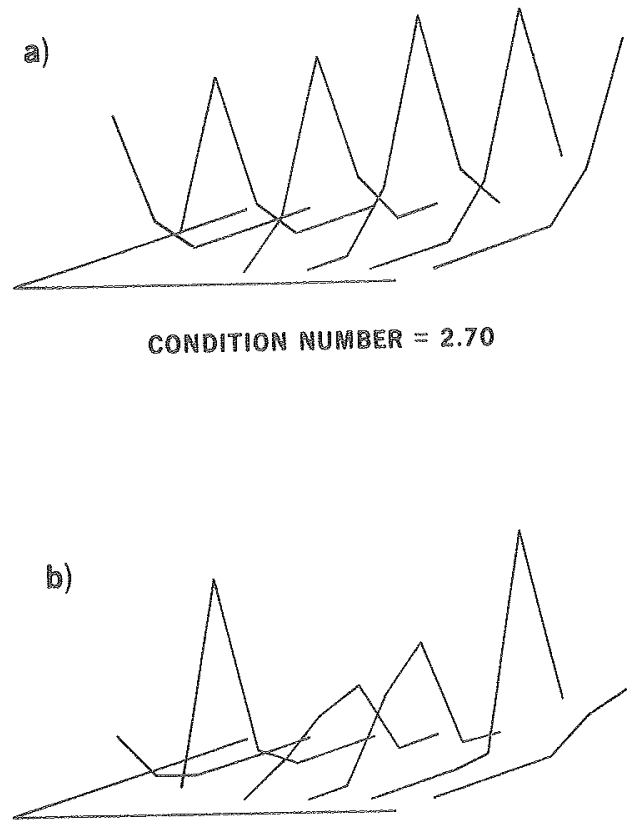

CONDITION NUMBER $=37.07$

XBL 788-9964

Fig. 2 (a) Values of a symmetric matrix $A^{\prime}$ for a onedimensional system of six points separated by $\Delta x=1 \mathrm{~cm}$. on a line in the geometrical center of a detector system. (b) Values of a matrix $A^{\prime}$ for $\Delta x=1 \mathrm{~cm}$. on a Tine displaced from the geometrical center of the detector sys tem resulting in a poorly invertible matrix.

This vector $\vec{r}_{2}$ contains as elements the relative rates detected by the $e^{\text {th }}$ coincidence when a point source is moved from the first to the last system point. Then the complete $\vec{k}^{\prime}$ due to a point source at $s_{j}$ will then be formed by

$$
\vec{k}^{\prime}\left(s_{j}\right)=\sum_{\ell} r_{\ell} \vec{r}_{\ell}
$$

which indicates that $\vec{k}^{\prime}\left(s_{j}\right)$ contains as elements a weighted sum of the relative rates detected by all possible coincidences generated by $s_{j}$ if a point source were to be moved from the first to the last system point. The structure of $\vec{k}^{\prime}$ contains, therefore, complete information about crosstalk, overlap or ambiguity between system points, as well as relative efficiency of its sampling by the detectors due to the scalar weights $r_{\ell}$.

Since for the case of unity activity at $s_{j}$ vector $\vec{k}^{\prime}$ is identical to the $j^{\text {th }}$ row of $A^{\prime}$, we can associate every row of $A^{\prime}$ to one system point, in the same order in which matrix $A$ was formed.
To illustrate these points, we shall consider two matrices $A^{\prime}$ generated by a positron-emitter imaging device, object of a companion paper, for two different sets of system points. Figure 2 a shows a perspective plot of the values of matrix $A^{\prime}$ for a system of six points with a very favorable condition number of 2.70. Six rows of six points each are plotted, joined by lines. If there were no overlap between detectors, only the $i^{\text {th }}$ point of the $i^{\text {th }}$ row would be different from zero. The finite size of the detectors results in each system point being partially coupled to the adjacent ones. Also, the first and last points are somewhat less well sampled than the other four. Figure $2 b$ shows the matrix $A^{\prime}$ for another system of rather unfavorable geometry with a high condition number of 37.07. Only the second and fifth points are clean, with little overlap. The third and fourth points are very strongly coupled and it would be very hard to distinguish between them in the presence of strong statistical fluctuations. The first and lastpoints are very poorly sampled by the detectors.

\section{Physical Meaning of Eigenvectors and Eigenvalues}

From the theory of matrices, we know that any vector corresponding to the nudimensional space spanned by a matrix can be expressed in terms of the eigenvectors of that matrix. In this manner, the experimentally determined vector $\vec{k}^{\prime}=A^{\top} k$ can be expressed as in Eq. 8,

$$
\vec{k}^{\prime}=\sum_{i} \alpha_{i} \vec{x}_{j}
$$

where

$$
\alpha_{i}=\left\langle\vec{k}^{\prime}, \vec{x}_{i}\right\rangle
$$

For the case under study, the eigenvectors $\vec{x}_{i}$ are arrays of melements, each element corresponding to a count rate at a system point (see Eq. 5 , for example). The eigenvectors $\vec{x}_{i}$, and therefore $\vec{k}^{\prime}$, can then be rewritten as functions of a space coordinate $r$, with the understanding that they are functions with discrete values only at system points.

Similarly, the values of $\alpha_{i}$ form a discrete function

in a space which we shall call frequency domain, by analogy with a Fourier-transformation. We can then rewrite Eqs. 15 and 16 as

$$
k^{\prime}\left(r_{j}\right)=\sum_{i} a\left(f_{i}\right) x_{i}\left(r_{j}\right)
$$

and

$$
a\left(f_{i}\right)=\sum_{j} k^{\prime}\left(r_{j}\right) x_{i}\left(r_{j}\right)
$$

which have the structure of a transform pair, i.e., $\alpha(f)$ is a transform of $k^{\prime}(r)$ in the m-dimensional Hilbert space of matrix $A^{\prime}$. The eigenvectors $\vec{x}_{i}$ form therefore a series of discrete functions analogous to sines and cosines in a Fourier-transformation. Figure 3 shows a set of eigenvectors for a one-dimensional system of 10 points obtained from measurements with the positron emitter imaging device. They are divided into approximately symmetric and antisymmetric functions about the center of the space, with an increasing frequency. 

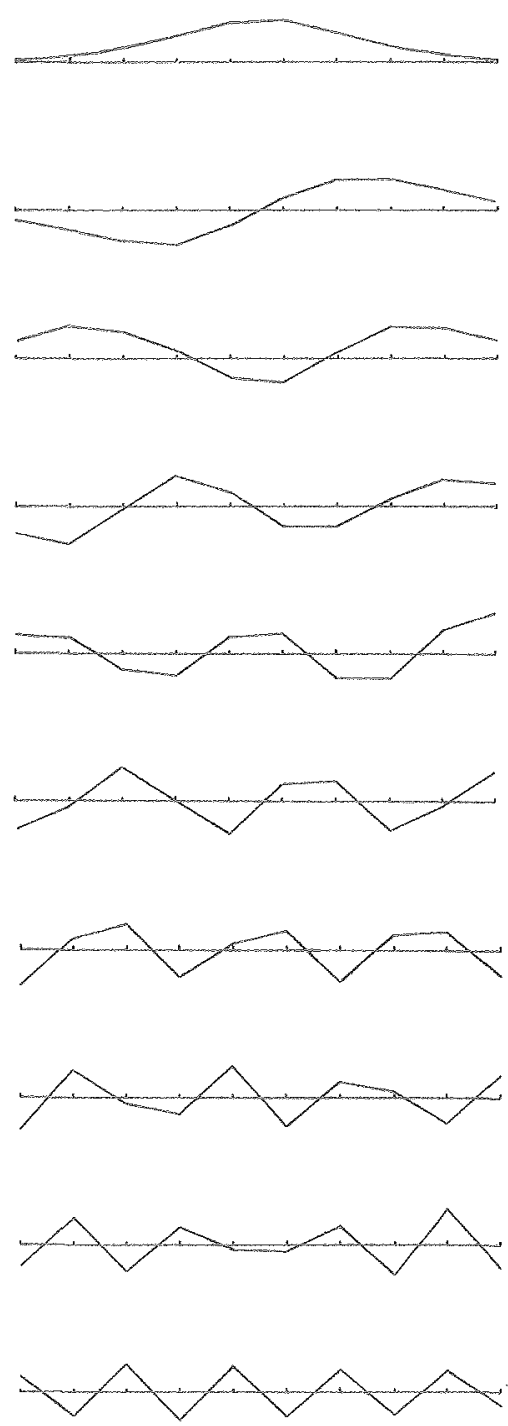

Fig. 3 A set of eigenvectors for a 10-point system in one-dimension.

Vector $\vec{k}^{\prime}$ is the result of a measurement which we still have to convert to $\vec{x}$ of $\mathrm{Eq} .1$ or 3 in order to solve for the unknown activity distribution. We can either solve the set of linear equations of Eq. 3 directly or we can use Eq. 10, which can be written as

$$
x\left(r_{j}\right)=\sum_{i} \frac{\alpha\left(f_{j}\right)}{\lambda\left(f_{i}\right)} x_{j}\left(r_{j}\right)
$$

The division in frequency domain by $\lambda\left(f_{i}\right)$ in going from $k^{\prime}\left(r_{j}\right)$ to $x\left(r_{j}\right)$ corresponds to a "deconvolution" in configuration space by a function which removes tube overlap and detector efficiency differences between system points. It must be pointed out that in the present case of generalized transforms the divisions $\alpha / \lambda$ may not correspond to a true deconvolution in configuration space, as would be the case with Fourier-transforms.
Relationship with Other Transforms

Image reconstruction from complete data sets by methods based on the Fourier-transforms are essen. tially an extension of the method discussed above for incomplete systems, particularly the method of deconvolution and back projection. Functions $x=e^{-i(\vec{\omega} \cdot \vec{r})}$ are used as eigenfunctions for the expansion of the results of a measurement, as in Eq. 17. This choice is fully justifiable as long as the imaging system has a space invariant point spread function."

The function $k^{\prime}(r)$ of $\mathrm{Eq}$. 17, when reconstructed, would be the result of a back projection without filtering. The process of deconvolution implied by the division of $\alpha / \lambda$ in frequency domain of $\mathrm{Eq} .19$ corresponds to the flltering necessary to remove the effects due to multiple rays determining the image of one point.

For incomplete data systems with a reasonable number of system points, the procedure that has been developed departs from the complete data case in that the set of orthogonal functions for the expansion and the eigenvalues for filtering are determined experimentally. Andrews and Hunt discuss the value of eigenanalysis in imaging space variant point spread functions.

Interpolation and Mathematical Sweeping of the

\section{Detector System}

The solution to Eq. I or 3 for a point source located exactly at one of the system points is a vector $\vec{x}$ with components which are all zero except for the one corresponding to the source position. If a point source is not located exactly at a system point, the response of the system cannot, in general, be predicted. It is reasonable to expect that the resulting vector $k$ will be some linear combination of the columns of matrix $A^{\prime}$ corresponding to the system points surrounding the position of the source. That is actually found to be the case in a real instrument if the system points are close to each other and the condition number of the matrix stays within some bounds. In such cases it is possible to interpolate between system points by using the Nyquist sampling theorem. In three dimensions, the theorem states that the best estimate that one can make of a function which is sampled at intervals $\Delta x, \Delta y, \Delta z$ is given by

$f(x, y, z)=$

$\sum_{j, j, k} g(i, j, k) \operatorname{sinc}\left(\frac{x}{\Delta x}-i\right) \operatorname{sinc}\left(\frac{y}{\Delta y}-j\right) \operatorname{sinc}\left(\frac{z}{\Delta z}-k\right)$

where $\operatorname{sinc}(x)=\sin (\pi x) /(\pi x), i, j, k$ are sampling point indices (system points), $g$ is the solution known at the system points and $f$ is the estimated function. The indices $i, j, k$ are zero at $x, y, z$ equal to zero.

Results of using the Nyquist interpolation procedure in one-dimension are shown in Figs. $4 a$ and b. A point source ( $\mathrm{Na}^{22}$ embedded in plastic) was positioned at $x=0$ and $0.75 \mathrm{~cm}$ between two groups of detectors (8 crystals each group) in the positron imaging device. Analysis was carried out with a matrix $A^{\prime}$ formed by 7 system points separated by $1 \mathrm{~cm}$ centered at $x=0$. Counting time was long enough to render statistical effects relatively small. The results of the matrix solution at the system points (horizontal scale lines with $\Delta x=1 \mathrm{~cm}$ ) are shown by fine lines. With the source at $x=0$ (or other system 
a)
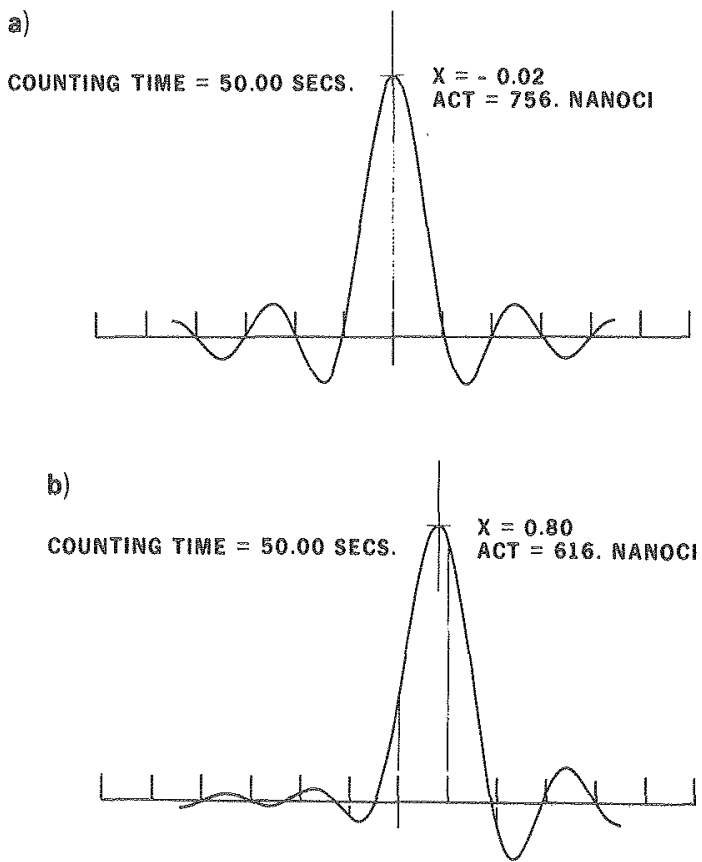

XBL 788-9967 points) position determination error is very small $(0.02 \mathrm{~cm}$ or less) and measured activity is quite consistent. Using the interpolation scheme for $x=$ $0.75 \mathrm{~cm}$, for example, position determination errors as large as $0.1 \mathrm{~cm}$ and a drop in detected activity are observed. The errors become smaller if the system points are brought closer, as for example with $\Delta x=$ $0.75 \mathrm{~cm}$. A7so, the detector positions and the number of detectors affect the accuracy of the interpolation scheme.

The solutions to Eq. 1 or 3 exhibit "ringing" at the system points in cases where the point source is not located at a system point. This effect is due to coupling between system points and is increased when $\Delta x$ is made smaller. In addition, the Nyquist images can magnify the "ringing." The theorem of Eq. 20 assumes that the source function $g$ does not contain frequencies above $(1 / 2 \Delta x) \mathrm{cm}^{-1}$ (in one dimension) and, therefore, no frequencies above that value are reproduced. This limitation in frequency (i.e., no sharp turns in the graph) and the requirement that the reconstruction be exact at the sampling points force the existence of the observed ringing magnification.

Another peculiarity of the method is the difa ferent width of the point response function with position, which makes further analys is of the images difficult.

Fig. 4 Performance of an $8 \times 8$ detector system for a $\mathrm{p}^{\mathrm{t}}$ - emitter point source positioned at $\mathrm{x}=0$ and $0.75 \mathrm{~cm}$, respectively. Reconstructed source positions and activities are shown next to the peaks (Nyquist interpolation).

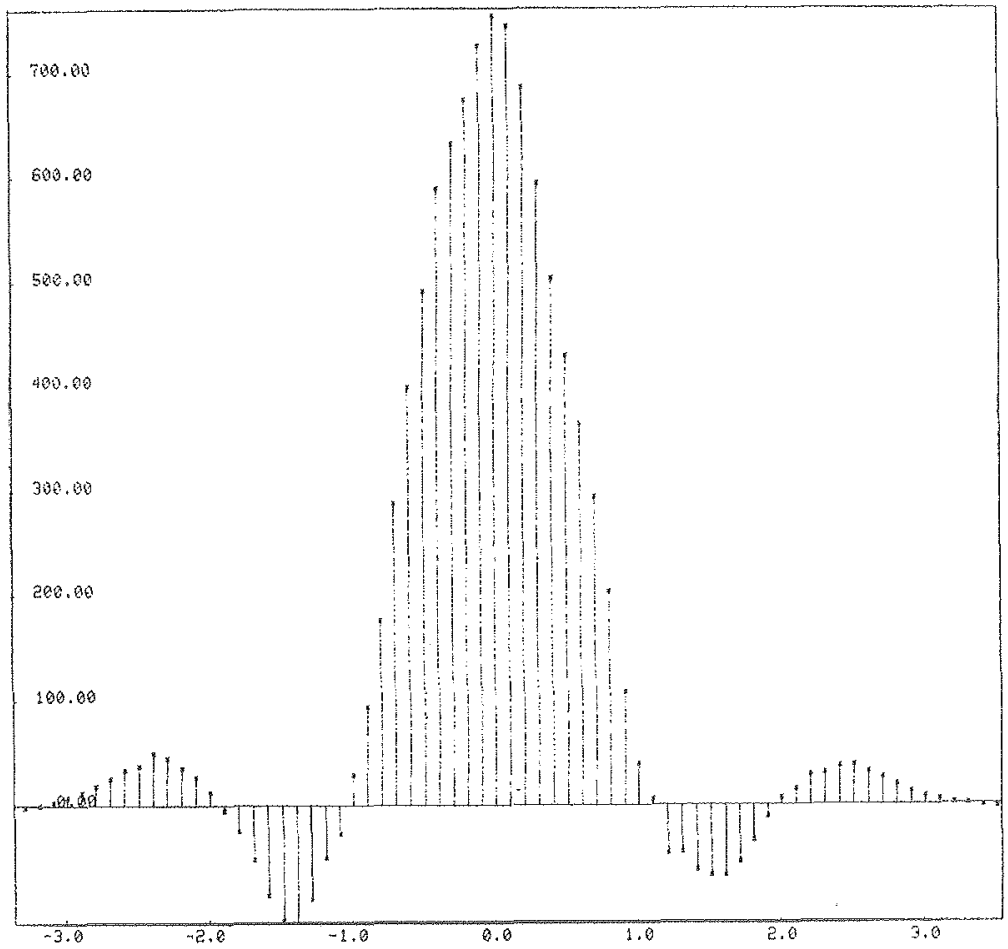

XBL 788-9975

Fig. 5 The same results of Fig. 5 analyzed by mathematical sweeping of the detectors. 
A common way of interpolation in imaging is by a mechanical sweeping of the detectors so that activities or densities corresponding to different sets of sampling points are obtained at different times. In the case of devices with detectors which overiap substantially in their coverage of the region to be imaged, sweeping can be done mathematically. This is carried out by analyzing successively an experimen. tally determined vector $k$ with matrices $A^{\prime}$ from sets of system points which have their origins displaced by small steps. As an example in one dimension, consider ten sets of system points with $\Delta x=1 \mathrm{~cm}$ and centers between $x=-0.4$ and $+0.5 \mathrm{~cm}$ in steps of $0.1 \mathrm{~cm}$. The experimental vector for the point source at $x=0$ which gave Fig. Aa has been analyzed in succession by the ten systems and the results super. imposed in Fig. 5. All the results analyzed show response functions with centroids within $\pm 0.05 \mathrm{~cm}$ of the correct values and the maxima, corresponding to detector activity, are much more consistent than in case of interpolation. Also, with exception of the tails, some systematic effects due to the detector configuration used, and minor statistical effects. the width and shape of the response function is invariant with source position, so that further image treatment by deconvolution, for example, appears more possible.

\section{Concluston}

This paper has studied the problems of imaing from a very limited number of projections as could arise, for example, from an imaging device which requires the use of a small number of large radiation detectors in some prescribed geometry. The theory developed has shown that the solutions to the imaging problem can be expanded into the set of eigenvectors of a system matrix which depends on the characteristics and geometry of the detector set. The method of solution is shown to lead to the conventional Fourier techniques in the limit of large numbers of totally independent detectors in a regular configuration. It has also been shown that observation of the system matrix and of its eigenvalues allows for a diagnosis technique that allows the determination of good useful detector configurations within some prescribed constraints. Finaliy, an interpolation technique using the sampling theorem and the concept of mathematical sweeping of the detector system is presented. The above concepts have been developed into the first version of an opera. tional instrument which is described in a companion paper.

\section{Acknowledgements}

The author would like to acknowledge the support of E. L. Appen, C. A. Tobias, F. S. Goulding and A. Chatterjee during the development of the project.

\section{References}

1. "Inage Processing for 2-D and 3-D Reconstruction from Projections: Theory and Practice in Medicine and the Physical Sciences," a digest of technical papers presented at Stanford University, Stanford, California, August 4-7, 1975.
2. J. S. Robertson, R. B. Marr, M. Rosenblum, V. Radeka and $Y$. L. Yamamoto, Tomographic Imaging in Nuclear Medicine, New York: Society of Nuclear Medicine, Inc., 1973.

3. J. H. Wilkinson, The Algebraic Eigenvalue Problem, Oxford: Clarendon Press, 1965.

4. H. C. Andrews and B. R. Hunt, Digital Image Restoration, New York: Prentice HaT1, 1977, Chap. 4. 

This report was done with support from the Department of Inergy. Any conclusions or opinions expressed in this teport represent solely those of the author(s) and not necessarily those of The Regents of the University of Callornia, the Lawrenee Berkeley I aboratory or the Department of Energy. 


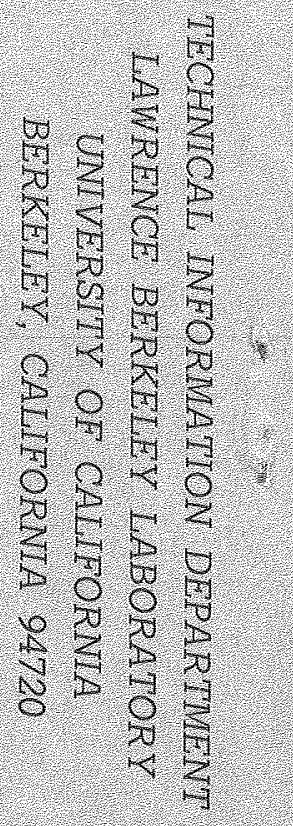

